Goldschmidt 2021 Abstract

https://doi.org/10.7185/gold2021.5465

\section{Joint Seismological-Geodynamical Assessment of Lower Mantle Temperature and Composition Variations}

\author{
JUN SU ${ }^{1}$, CHRISTINE HOUSER ${ }^{1}$, JOHN HERNLUND ${ }^{1}$ AND \\ FREDERIC DESCHAMPS ${ }^{2}$ \\ ${ }^{1}$ Tokyo Institute of Technology \\ ${ }^{2}$ Academia Sinica \\ Presenting Author: junsu@elsi.jp
}

Uncorrelated seismic $\mathrm{P}$ - and S-wave velocity $\left(\mathrm{V}_{\mathrm{P}}\right.$ and $\left.\mathrm{V}_{\mathrm{S}}\right)$ variations in the Earth's mantle are indicative of non-thermal heterogeneity, such as variations in phase and/or composition. Seismic tomography reveals a discrepancy between $V_{P}$ and $V_{S}$ below $\sim 2200 \mathrm{~km}$, which has been used to argue for the existence of large-scale heterogeneity in chemical composition. Nevertheless, some uncertainties remain regarding the relative resolving power of $\mathrm{V}_{\mathrm{P}}$ and $\mathrm{V}_{\mathrm{S}}$ tomography models, and purely thermal models (i.e., without chemical heterogeneity) have not been entirely excluded. Here we use a singular value decomposition of two mutually consistent $\mathrm{V}_{\mathrm{P}}$ and $\mathrm{V}_{\mathrm{S}}$ models to construct a "tomographic filter" and apply it to the outcomes of mantle convection models for both purely thermal and thermalchemical layering scenarios. This is the first time such a procedure has been performed with both a $V_{P}$ and $V_{S}$ model. A filtered lower mantle model with only temperature variations does not yield a large low $\mathrm{V}_{\mathrm{S}}$ anomaly, and is inconsistent with seismic observations. By contrast, the filtered model predicted using both composition and temperature variations results in a slow $\mathrm{V}_{\mathrm{S}}$ anomaly and a neutral $\mathrm{V}_{\mathrm{P}}$ anomaly, which provides a good correspondence with observations. A dense (Fe-rich), high bulk modulus, and low shear modulus may explain the seismic observations and provide for geodynamical stability against entrainment by mantle convection. Such dense "chemical piles" or other structures have been attributed to the accumulation of basaltic crust, core-mantle interactions, cumulates of a basal magma ocean, and/or residues of early differentiation.
Synthetic $V_{p}$ anomaly (\%)

for temperature, composition, and post-perovskite

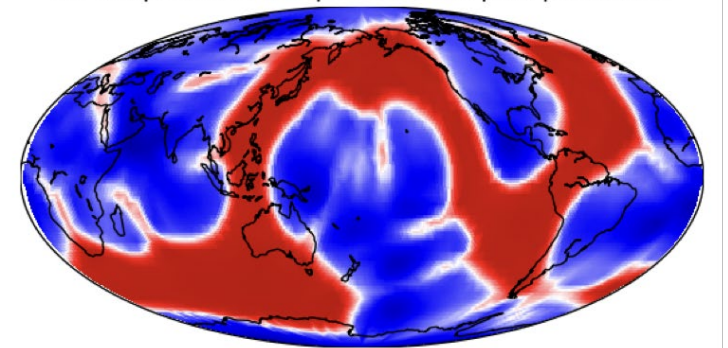

$V_{p}$ anomaly model (\%) after tomographic filter

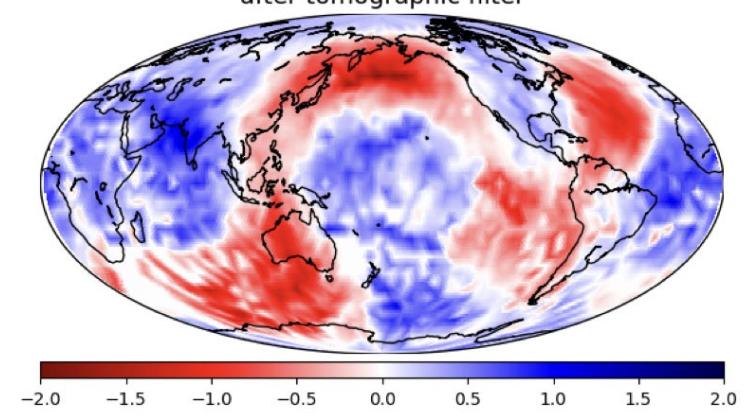

